In Cres. Vol. $4 N^{\circ}$ 2: pp. 293-303, 2013

\title{
LA ADMINISTRACIÓN FINANCIERA Y SU INCIDENCIA COMO ELEMENTO INTEGRADOR EN LOS GOBIERNOS LOCALES DE LA REGIÓN LA LIBERTAD*
}

\author{
FINANCIAL MANAGEMENT AND ITS IMPACT AS AN INTEGRATOR \\ SYSTEM IN THE LOCAL GOVERNMENTS OF LA LIBERTAD
}

María Antonia Campos Vargas ${ }^{1}$

\begin{abstract}
RESUMEN
La presente investigación tiene por objeto buscar la interrelación de los sistemas de administración financiera del Estado como elemento integrador en los gobiernos locales de la Región La Libertad, periodo 2011-2012, aplicando controles internos, para que la utilización de los recursos públicos sea transparente.

El problema que nos planteamos es ¿De qué manera una adecuada administración financiera como elemento integrador de los sistemas administrativos repercutió en los gobiernos locales en el periodo 2011-2012?

La hipótesis que se formuló es que la administración financiera gubernamental como elemento integrador, incide en los gobiernos locales, porque nos permite una mejor y oportuna eficacia, eficiencia y economía para el logro de objetivos y métodos institucionales

Los ámbitos de estudio utilizados fueron la Municipalidad Distrital de La Esperanza, distrito de la provincia de Trujillo, Región la Libertad, así como la Municipalidad Distrital de Paijan, provincia de Ascope.

El método que se utilizó fue el analítico, inductivo y deductivo, y las técnicas: la entrevista, observaciones directas, consulta a expertos y el acervo documentario de dichas municipalidades.
\end{abstract}

* Recibido: 19 de junio del 2013; aprobado: 02 de noviembre del 2013.

1 Contador Público Colegiado. Universidad Nacional de Trujillo. Administradora de la Municipalidad distrital de La Esperanza de la provincia de Trujillo - Perú 
Como resultado, podemos afirmar que una adecuada administración financiera como elemento integrador de los principales sistemas administrativos, como presupuesto, contabilidad, abastecimiento, tesorería y recursos humanos, nos permite la transparencia y el buen uso de los recursos ordinarios, recursos directamente recaudados, endeudamiento, donaciones y transferencias, así como de los recursos que maneja el Tesoro Público en el logro de las metas.

PALABRAS ClAVE: Administración financiera, Sistemas Administrativos, Gobiernos Locales.

\section{ABSTRACT}

The present research aims of study search the interrelationship of systems of financial administration of the State as an integrating element in the local governments of the La Libertad Region, period 2011-2012, applying internal controls, to make the use of public resources transparent. The problem to investigate is the following how a proper financial administration as Integrator of administrative systems, impact on Governments in the period 2011-2012 the hypothesis that was made is that the governmental financial administration as Integrator, has an impact on local governments, because it allows us a better and timely effectiveness, efficiency and economy for the achievement of objectives and institutional methods used study material and that serves as a sample is? the municipality district of the hope district of the province of Trujillo, the Libertad Region, as well as the municipality Paiján district, province of Ascope method that was used was the analytical, inductive and deductive and techniques the interview, direct observations, consulting experts and heritage documentary of these municipalities as result can confirm that financial administration as Integrator of the main administrative systems such as budget, accounting, supply and Treasury and human resources, a proper allows us to transparency and the proper use of regular resources Resources directly collected, indebtedness, donations and transfers, as well as of resources handled by the public Treasury in the achievement of the goals.

KEY WORDS: Financial management, Systems administrative, Local governments.

\section{INTRODUCCIÓN}

La gestión financiera gubernamental es una secuencia de la aplicación de un conjunto de principios, normas, organismos, recursos, sistemas y procedimientos que intervienen en las operaciones de programación, gestión, registro, información y control de los fondos públicos, a través de la captación de ingresos y ejecución de gastos armonizados en un presupuesto previamente predeterminado y encausado al logro de objetivos y metas del estado a través de las entidades e instituciones públicas que lo conforman para el desarrollo de la sociedad. 
Para la administración de los fondos públicos se ha constituido sistemas administrativos, que tienen por finalidad regular la utilización de los recursos en las entidades de la administración pública promoviendo la eficacia y la eficiencia en su uso.

Estos sistemas administrativos de aplicación nacional están referidos a las siguientes materias: gestión de recursos humanos, abastecimientos, presupuesto público, tesorería, endeudamiento público, contabilidad, inversión pública, planeamiento estratégico, defensa judicial del estado, control y modernización de la gestión pública.

Los sistemas administrativos estarán a cargo de un ente rector que se constituye en su autoridad técnico-normativa a nivel nacional; dicta las normas y establece los procedimientos relacionados con su ámbito; coordina su operación técnica y es responsable de su correcto funcionamiento en el marco de la ley $\mathrm{N}^{\circ} 28112$ denominada: LEY MARCO DE LA ADMINISTRACIÓN FINANCIERA DEL SECTOR PÚBLICO (Ley SIAF). Esta ley establece normas básicas para lograr una gestión integral eficiente en el proceso de captación y utilización de los fondos públicos, contribuyendo de esta manera al cumplimiento de los deberes y funciones del Estado.

Los sistemas que integran esta ley marco de la administración financiera del Estado son: Sistema Nacional de Presupuesto, Sistema Nacional de Tesorería, Sistema Nacional de Endeudamiento, Sistema Nacional de Contabilidad.

El Sistema Integrado de Administración Financiera (SIAF) es un sistema de registro automatizado de operaciones y procesamiento de la información de las transacciones que se realizan durante el proceso de ejecución financiera y presupuestal, que tiene por finalidad centralizar la información que se genera en los subsistemas de presupuesto, tesorería y contabilidad, con el objetivo de sustituir, con el apoyo de la tecnología, todo tipo de información que fluye en cada unidad ejecutora hacia cada órgano rector (Economía y Finanzas, Tesoro Público, Dirección Nacional de Contaduría Pública, SUNAT, Contraloría General de la Republica, Congreso de la Republica, etc.), mediante un registro único de operaciones con mayor rapidez, precisión e integridad de informes.

Para ello se ha creado una base de datos cuya sede central se encuentra en el Ministerio de Economía y Finanzas, contando con una operatividad con 580 computadoras distribuidas a nivel nacional, para cada una de las unidades ejecutoras y en 1826 municipios. 
SIAF- Presupuesto de la Base de Datos del SIAF, ubicado en el Ministerio de Economía y Finanzas, está expresado en un lenguaje ORACLE y el lenguaje se emplea en las unidades ejecutoras que se interconectan con el SIAF y se expresa en el lenguaje Visual Basic.

Se encarga de centralizar la información y registro único de operaciones que se generan en los subsistemas (incluido el Área de Abastecimientos) con la finalidad de acelerar los procesos de registro, control de datos y obtener información en línea oportuna, precisa y clara, que fluya de cada unidad ejecutora hacia los órganos rectores (Ministerio de Economía y Finanzas, Tesoro Público, Dirección Nacional de Contaduría Pública, SUNAT, OSCE, Superintendencia de Bienes Nacionales, etc.) para la toma de decisiones sobre la base de los informes emitidos, referidos al resultado de las operaciones financieras y presupuestales, tales como: formulación y sus respectivas modificaciones presupuestarias, ejecución presupuestal, contabilización de las operaciones financieras y recaudación y registro de ingresos.

\section{APLICACIÓN DEL SIAF EN LOS GOBIERNOS LOCALES}

Es de aplicación en todas las municipalidades del país en concordancia con lo dispuesto por el artículo 10 de la Ley 28112, Ley marco de administración financiera del sector público, "El registro de información es único y de uso obligatorio por parte de todas las entidades y organismos del sector público a nivel nacional, regional y local, y se efectúa a través del SIAF, que administra el MEF, a través del comité de coordinación”.

\section{EL SIAF-GL}

El Sistema Integrado de Administración Financiera - GL es una herramienta que permite ordenar la gestión administrativa de los gobiernos locales; simplificar considerablemente sus tareas y reducir los reportes que se elaboraban, así como el tiempo dedicado a la conciliación.

El sistema recoge la normatividad vigente de cada uno de los órganos rectores y de control y promueve las buenas prácticas, el uso eficiente de los recursos públicos, la rendición de cuentas y la transparencia.

El SIAF-GL cuenta con los siguientes módulos: Presupuestal (Formulación, Modificaciones, Asignación Mensual); Ejecución Presupuestal - Tesorería (Administrativo); y Registro de Recaudación. 


\section{MODULO PRESUPUESTAL}

Es el proceso a través del cual se registra el presupuesto inicialmente aprobado llamado PIA, así como las modificaciones efectuadas durante el ejercicio (PIM). Se registran también las cadenas funcionales programáticas y los clasificadores de ingreso y gasto en: PIA (Presupuesto Institucional de Apertura), PIM (Presupuesto Institucional Modificado) y Cadena Funcional Programática.

\section{MÓDULO DE EJECUCIÓN PRESUPUESTAL - TESORERÍA}

El Módulo de Ejecución Presupuestal - Tesorería (Administrativo) está organizado en 2 partes: Registro Administrativo: Ejecución de ingresos y Ejecución de gastos y Registro Contable: Contabilización de las fases, así como de las notas de contabilidad para la obtención de los estados financieros y presupuestarios, de acuerdo a los instructivos $\mathrm{N}^{\circ} 6$ y 7 de la Contaduría Pública de la Nación.

\section{REGISTRO ADMINISTRATIVO}

Ejecución de ingresos. La ejecución de los fondos públicos se produce cuando la entidad (o el Estado) determina o recauda, capta u obtiene los recursos financieros efectivamente, los mismos que serán destinados a financiar los gastos previstos. Comprende las etapas de: determinación, recaudación, captación y obtención.

Ejecución de gastos. La ejecución del gasto público abarca los desembolsos efectuados por concepto de gastos corrientes, capital o inversión pública, así como el pago de obligaciones por deuda pública. Cada mes los gobiernos locales generarán en SIAF-GL, su Calendario de Compromisos, que establece los topes máximos de gasto de dicho mes, para la ejecución del gasto público. Comprende las fases de: compromiso, devengado, girado, pagado.

\section{REGISTRO CONTABLE}

Es el proceso básico para el registro de las operaciones que realiza la entidad, permitiendo un registro único de contabilización, así como su acoplamiento modular, asegurando su procesamiento automático de la información.

Se contabilizan los ingresos (fase de determinado y recaudado) y los egresos (compromiso, devengado, girado y pago) utilizando la Tabla de Operaciones (TO SIAF), matriz que relaciona los clasificadores presupuestales con las cuentas del Nuevo Plan Contable Gubernamental. 
El SIAF tiene por finalidad agilizar los procesos de los registros a través del sistema mediante computadoras instaladas a nivel nacional por el MEF, otorgando a cada municipalidad un equipo completo para poder tener la información interconectada, controlando y procesando los datos, obteniéndose información en tiempo real, oportuno, claro y preciso, información que se utiliza para la toma de decisiones a través de informes contables resultantes de las operaciones financieras y presupuestales.

\section{PROBLEMA}

¿De qué manera la administración financiera como elemento integrador incidió en los gobiernos locales de la Región La Libertad en el periodo 20112012 ?

\section{HIPÓTESIS}

La administración financiera como elemento integrador incidió de manera positiva en los gobiernos locales de la Región La Libertad - periodo 2011-2012.

\section{OBJETIVO GENERAL}

Determinar la interrelación y la incidencia de los sistemas de administración financiera del Estado con los controles internos y externos, con el objeto de incrementar la eficacia de éstos para generar una adecuada efectividad entre el gobierno central, gobiernos regionales y gobiernos locales, promoviendo así la transparencia, integración, equidad y optimización de los recursos.

\section{OBJETIVOS ESPECÍFICOS}

1. Ejecutar los recursos públicos en un marco de absoluta transparencia.

2. Generar información en línea, oportuna y confiable para la toma de decisiones.

3. Propiciar en los gobiernos regionales y locales el acceso a las nuevas tecnologías de información y comunicación electrónicas.

4. Integrar a los gobiernos regionales y locales mediante el uso de medios tecnológicos y electrónicos en sus diferentes niveles.

5. Mejorar el nivel de conocimiento de los responsables de las áreas de actividad o de los sistemas administrativos, logrando una mejora sustancial de la calidad de la gestión pública. 
6. Propiciar la transparencia de la información en la gestión pública asegurando el correcto uso de los fondos públicos.

\section{UNIVERSO Y MUESTRA}

El universo de estudio comprendió todos los gobiernos locales de la Región La Libertad. La muestra estuvo conformada por las municipalidades distritales de La Esperanza, provincia de Trujillo y Municipalidad Distrital de Paiján, provincia de Ascope, Región La Libertad, años 2011-2012.

\section{MATERIALES Y MÉTODOS}

Se utilizó el método analítico, inductivo y deductivo, así como el método descriptivo y normativo. Y como técnicas se aplicaron las observaciones directas, consulta a expertos y acopio documental de dichas municipalidades.

El diseño de investigación utilizado fue el diseño clásico porque se tomó como grupo experimental la Municipalidad Distrital de La Esperanza y como grupo testigo de control la Municipalidad Distrital de Paiján.

\section{RESULTADOS}

La administración financiera engloba la totalidad de las rentas y los gastos públicos, cualesquiera sea su naturaleza, origen o destino. Por lo tanto, abarca rentas y gastos de todas las instituciones del sector público, sean estas centralizadas, descentralizadas, autónomas, regionales o seccionales.

Una característica básica del sistema de contabilidad es que participa como componente integrador de los datos y la información financiera en los gobiernos locales.

Los sistemas de administración del Estado tienen como finalidad estructurar una solución que sirva de guía o modelo para el diseño y organización del sistema de administración financiera y control interno aplicable en las entidades del sector público.

Los resultados de esta investigación se fundamentan en las experiencias positivas obtenidas en Bolivia, Argentina, El Salvador, Panamá, Venezuela, Paraguay, Nicaragua, Honduras, Colombia y Guatemala, con una estrategia de acción y un enfoque sistémico, promoviendo la eficacia, eficiencia y economía en el uso de dichos recursos.

La administración de los recursos financieros es uno de los sistemas adjetivos y de apoyo a los gobiernos locales y, por ende, a la administración pública. 
La administración financiera como sistema planifica, obtiene, asigna, utiliza, registra información y control interno, persigue la eficiente gestión de los recursos financieros del Estado para la satisfacción de las necesidades colectivas.

Los componentes del macrosistema de administración financiera son los siguientes:

Gestión de Recursos Humanos, Abastecimiento, Presupuesto Público, Tesorería, Endeudamiento Público, Contabilidad, Inversión Pública, Planeamiento Estratégico, Defensa Judicial del Estado, Control, Modernización de la gestión Pública.

En el gráfico se observa el elemento integrador de la Administración Financiera en los gobiernos locales, regionales y nacionales, a nivel descentralizado y a nivel central.

Estos componentes disponen de sus correspondientes controles internos y todos ellos, en sus distintos momentos, se encargan de prever, procesar e informar sobre las operaciones relacionadas con el manejo de los recursos financieros públicos. Cada sistema funciona en base de insumos que se procesan y producen flujos de información dirigidos a los niveles gerenciales para orientar y respaldar la toma de decisiones

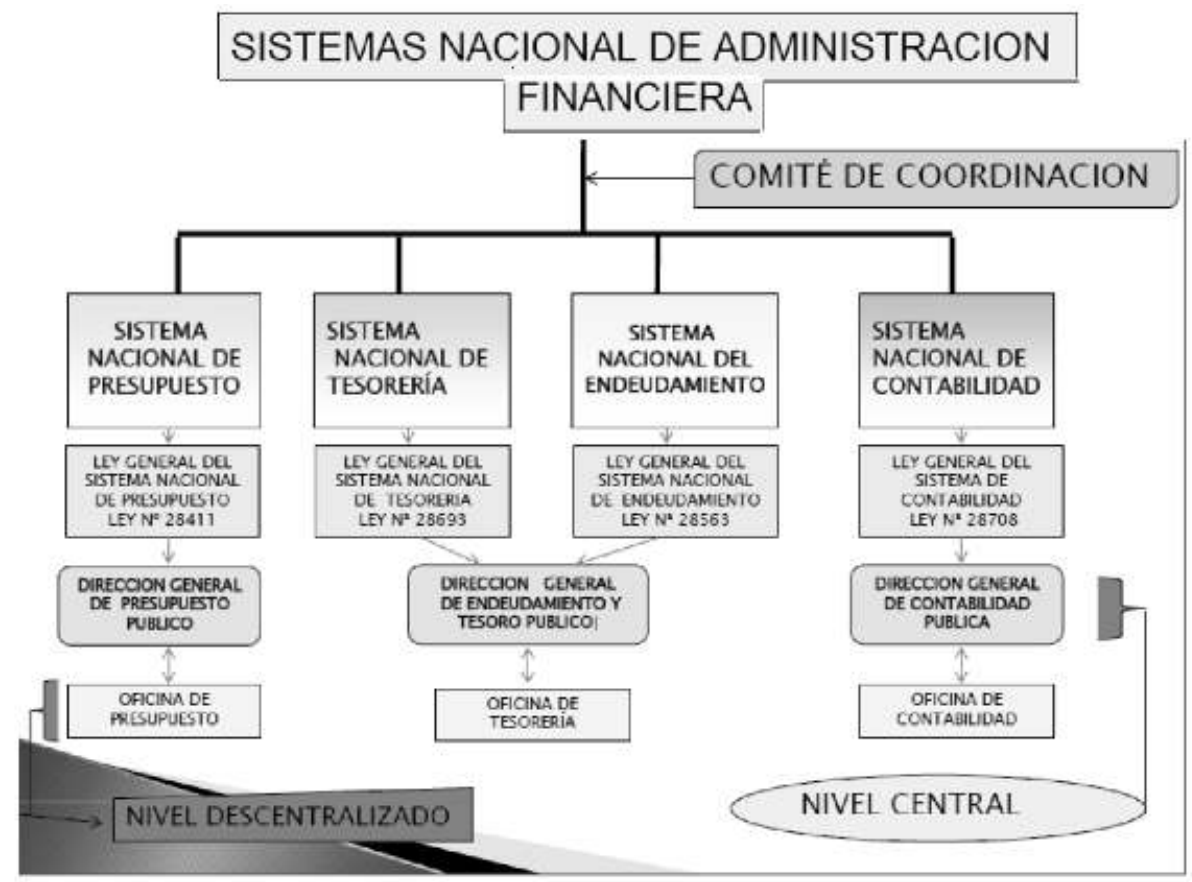




\section{EL SIAF Y SUS VINCULACIONES CON LOS GOBIERNOS LOCALES}

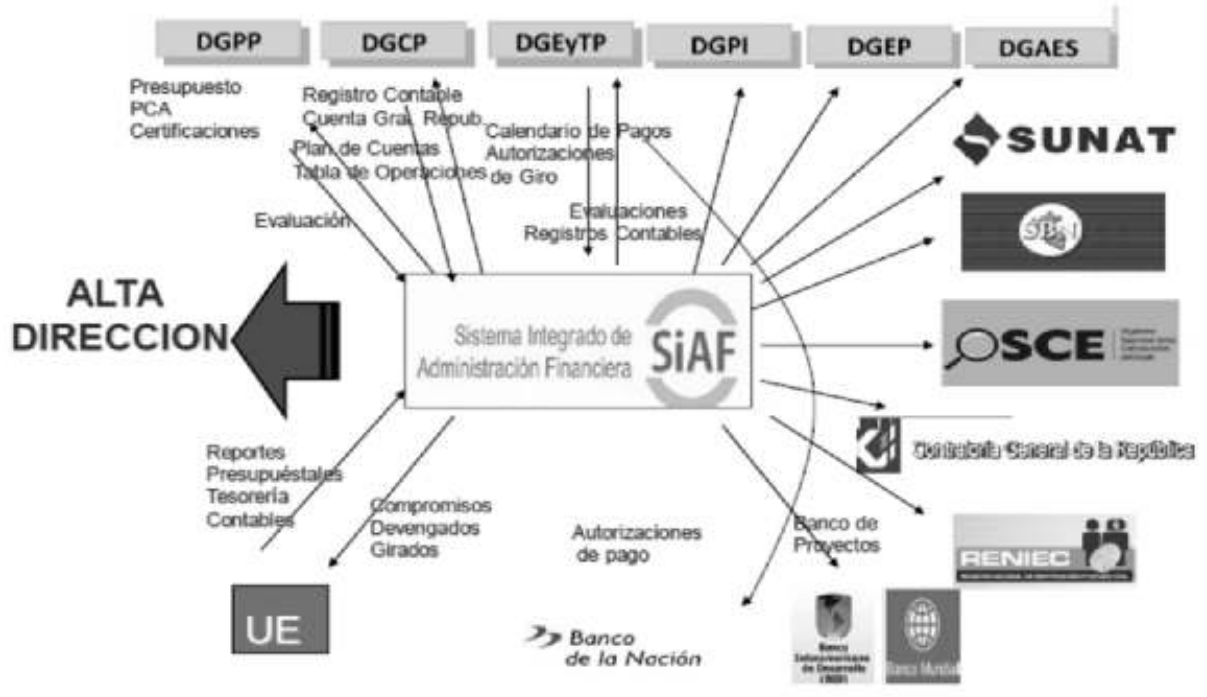

\section{DISCUSIÓN}

Los componentes del sistema de administración financiera no deben ser considerados aisladamente; pues, están ligados entre sí, formando una unidad conceptual indisoluble en virtud del fin único que los engloba. La previsión aplicada al presupuestar, cuando esta se ejecuta, se traduce en rentas y gastos que se registran e informan en diferentes instancias del proceso seguido por las transacciones financieras en los sistemas de presupuesto, tesorería y contabilidad.

Una característica básica del sistema de contabilidad es que participa como componente integrador de los datos y la información financiera, al consolidar su información financiera y presupuestaria, dando como resultado el Estado de Situación Financiera en los Gobiernos locales.

La administración financiera según los resultados, engloba la totalidad de los ingresos por toda fuente de financiamiento y recurso y de los gastos públicos; por lo tanto, abarca rentas y gastos de todas las instituciones del sector público, sean estas centralizadas, descentralizadas, o autónomas.

Del análisis podemos afirmar que la característica principal del sistema de administración financiera en el ámbito descrito es la centralización normativa y la descentralización operativa, conjunto de medidas y métodos adoptados y 
aplicados por cada institución en el cumplimiento de sus ineludibles obligaciones gerenciales; y tiene por objeto salvaguardar los recursos, garantizar la confiabilidad y corrección de las operaciones y de la información producida, estimular la adhesión a las políticas y normas establecidas, promover la eficiencia y economía en el uso de los recursos y fomentar la efectividad en el logro de las metas y objetivos.

La función gerencial está dirigida a la obtención de los mejores resultados con el menor uso de recursos y esfuerzos. Con este propósito, la gerencia adopta sus decisiones garantizadas en la verificación y validación de las operaciones, mediante los procedimientos de control interno.

\section{CONCLUSIONES}

1. La información financiera y presupuestaria con el sistema de contabilidad tradicional hasta el 2005 no era consistente y relevante por cuanto no brindaba un reporte integral de la gestión administrativa financiera; pero a partir del 2006, con la implementación del SIAF como elemento integrador y el uso adecuado de los sistemas administrativos, la gestión municipal es más eficiente y eficaz.

2. La administración financiera como elemento integrador en los gobiernos locales de la Región La Libertad, permite optimizar el uso de los recursos públicos en objetivos y metas institucionales programadas.

3. El presupuesto público de los gobiernos locales permite controlar la programación y ejecución del gasto, a fin de que se cumplan los principios de transparencia, legalidad, objetividad y optimización de los recursos públicos.

4. Una administración financiera integral permite mejorar el nivel de conocimiento del personal y funcionarios responsables de las áreas administrativas, así como velar por el buen uso de los recursos.

5. Una adecuada administración financiera como elemento integrador de los sistemas administrativos permite racionalizar los gastos corrientes y gastos de capital en un gobierno local o municipal. 


\section{BIBLIOGRAFÍA CONSULTADA}

Alvarado Mairena, J. (2005) Contabilidad Gubernamental. Centro Interamericano de Asesoría Técnica. CIAT Perú.

Alvarez IlaneZ, J.(2001) Formulación de Estados financieros y Presupuestarios en el Sector Publico y Gobiernos locales. Editorial y Distribuidora Real SRL.

Ayala Zavala, P. (2009) Manual para la Aplicación de Nuevo Plan Contable Gubernamental. Ediciones y Distribuciones Palma. Lima.

Bellido SANCHEZ, P. (2005) Administración Financiera. Editorial Técnico Cientifica SA. Lima.

Contaduría Publica de la NACión (1998) Manual de contabilidad Gubernamental de la Municipalidades, Lima-Perú.

Contraloría General DE la RepúBlica (2003) Manual de Auditoria Gubernamental. Editora Perú. Lima.

Directiva de Tesorería No 001-2011-EF/51.01.

Ley Marco de Administración Financiera del Sector Público - Ley Nº 28112 (Ley SIAF).

Ley $\mathrm{N}^{\circ} 27444$ Ley de Procedimientos Administrativos.

Ley N²9812, Ley del Presupuesto para el Sector Publico para el año fiscal 2012.

Ley $N^{\circ} 27972$ Ley Orgánica de Municipalidades.

Resolución Directoral Nº09-2012-EF/95.01 - Prorrogar hasta el 30 de junio del presente año, el plazo de presentación de la información financiera y presupuestaria del primer trimestre del ejercicio 2012.

Resolución Directoral $N^{\circ}$ 011- 2012-EF/95.01 Aprobar la Directiva $N^{\circ}$ 002-2012-EF/51.01 Preparación y presentación de la información financiera, presupuestaria y complementaria trimestral y semestral por las empresas y entidades de tratamiento empresarial del estado.

RD. $N^{\circ}$ 002-2012-EF/51.01.

VAn HoRne, J. (2005) Administración Financiera. Litográfica. Ingramex SA. México. 